\title{
LA ADMINISTRACIÓN DE ARCHIVOS: UNA PROPUESTA DE PROFESIONALIZACIÓN
}

\author{
Georgina Flores Padilla*1 \\ Celia Ramírez López*2 \\ Felipe Ramírez Peña*3 \\ César Augusto Ramírez Velázquez*4 \\ Sandra Peña Haro*5
}

\begin{abstract}
Resumen
En México los archivos públicos y privados son frecuentemente dirigidos por personal con formación empírica, lo que debilita la administración y gestión. El sistema de educación superior de México tiene dos opciones en el campo archivístico, aunque ninguna de las carreras ofrece como un elemento central la administración. Por lo anterior, no se están preparando profesionales con el perfil que requieren las instituciones y organizaciones para el manejo documental y de la información, tareas vitales para tomar decisiones y preservar la memoria. Así, se propone la creación de una licenciatura que considere como uno de sus ejes la administración de archivos y la archivística, enmarcada por las ciencias sociales y humanidades.
\end{abstract}

Palabras clave: licenciatura, administración de archivos, gestión documental.

\section{Abstract}

In Mexico non-specialists run a large number of public and private archives, which weakens the administration and management. The higher education system in Mexico provided is limited since there are only two options that offer a degree in Archival Science. None

${ }^{* 1}$ IISUE- UNAM.

$*^{2}$ IISUE- UNAM.

*3 FFyL- UNAM.

$*^{4}$ IIBI-UNAM.

${ }^{* 5}$ IISUE- UNAM. 
of these options offer management as the fundamental axis of the professional training, such situation leads to insufficient professional profiles. The institutions and organizations require graduate specialist in document management and information tasks, vital knowledge for the decision-making and preservation of memory. Therefore, this paper develops a proposal for a bachelors degree that includes, as core variables of the program, management and archival science topics framed by humanities and social sciences.

Keywords: higher education, management and archival science.

\section{Presentación}

Los archivos resguardan los documentos que conforman la memoria sobre el quehacer de diversas áreas del conocimiento humano. Las transformaciones actuales han generado cambios en los paradigmas de la administración de archivos.

La publicación de una normativa federal en materia de archivos y acceso a la información ha incrementado las exigencias para los responsables y ha subrayado la importancia de la profesionalización. Por lo anterior, se ha experimentado un paulatino y sensible aumento en la demanda de profesionales con características que no se contemplan en los planes de estudios en archivística que se imparten en el país.

Al proponer la licenciatura en administración de archivos y gestión documental se consideró que es momento de consolidar una profesión necesaria para el país, pues su alcance laboral es amplio y con una proyección a corto, mediano y largo plazo. También se apreció que los egresados deberán estar en formación permanente, y considerar la posibilidad de ingresar al posgrado, que dará a la profesión mayor solidez académica. Es importante mencionar que en una disciplina emergente como la archivística, existe un potencial amplio de temas y líneas de investigación que posibilitan el desarrollo de proyectos pertinentes y originales que ayudarán a resolver problemas en las instituciones y organizaciones. 


\section{Antecedentes. Los afanes del Estado mexicano. De la Biblioteca Nacional a la Secretaría de Educación Pública}

En 1915 Agustín Loera y Chávez, como Subdirector de la Biblioteca Nacional, observó la necesidad incrementar los conocimientos de quienes procesaban los libros y documentos, de ahí concibió la idea de fundar la Escuela Nacional de Bibliotecarios y Archiveros, cuyo proyecto presentó al gobierno el 7 de julio de ese año. ${ }^{1}$

En la planeación se acordaron ciertos puntos, dentro de los cuales destacan que la escuela quedaría adscrita a la Biblioteca Nacional, los estudios serían teórico prácticos, durarían un año y tendrían un carácter gratuito. ${ }^{2}$ Por su parte, los requisitos de ingreso para los alumnos serían contar con educación primaria y tener entre 15 y 45 años de edad. La asistencia al primer curso sería obligatoria para los empleados de las bibliotecas y archivos del Distrito Federal y para aquellos que quisieran ocupar los puestos vacantes. ${ }^{3}$

Aprobado el proyecto, se inauguró la Escuela Nacional de Bibliotecarios y Archiveros en el salón de cátedras de la Biblioteca Nacional, el 24 de junio de 1916. ${ }^{4}$ La nueva institución quedó bajo la dirección de Loera y Chávez. El primer curso tuvo una duración de un año, como se tenía planeado, y contó con 121 alumnos. ${ }^{5}$

El segundo curso para bibliotecarios y archiveros se realizó de 1917 a 1918 con una inscripción de 114 alumnos. La administración determinó ampliar el curso a dos años, además se creó la Academia de Bibliografía en la cual los alumnos realizarían sus prácticas. ${ }^{6}$

1 Iguíniz, Juan Bautista "Apuntes para la historia de la enseñanza de la Biblioteconomía en México, p. 13.

2 Sotelo Linares, Sandra, "Panorama de la enseñanza de la Bibliotecología en la UNAM. Informe Académico por elaboración comentada de Material didáctico para apoyar la docencia”, p. 13. 3 Ibidem, p. 13.

4 Villanueva Bazán, Gustavo, "La Archivística: una ciencia en busca de sí misma (México 1915-1945)", p. 31.

5 Sotelo, "Panorama...", op. cit., p. 13. La autora nos dice que las asignaturas fueron: clasificación de bibliotecas y archivos; organización de bibliotecas y archivos; catalografía; traducción de francés; traducción de inglés; traducción del latín y conferencias de bibliología. 6 Villanueva, "La Archivística...", op. cit., p. 47. El autor agrega que hubo cambios en la currícula: paleografía y latín se impartirían en dos cursos anuales; así como, francés durante el primer año e inglés solo el segundo. p. 47. 
Con una inscripción de 32 alumnos se abrió el tercer curso el 4 de marzo de 1918, sin embargo, la escuela se cerró el 17 de mayo del mismo año por órdenes de Venustiano Carranza, quien argumentó las difíciles condiciones del erario nacional. ${ }^{7}$

En la década de los treinta del siglo xx se impartieron cursos a iniciativa de Francisco Gamoneda, -destacado bibliotecario y archivista, quien daría impulso a los estudios de archivonomía en México- éstos cursos se impartieron en 1931. De 1937 a 1938 se efectuó un curso de biblioteconomía y archivonomía en la Hemeroteca de la Secretaría de Hacienda, dictado nuevamente por Gamoneda. ${ }^{8}$

Después del tercer congreso de bibliotecarios y primero de archivistas, efectuado en 1944 se concretó la creación de la Escuela Nacional de Bibliotecarios y Archivistas, dependiente de la Secretaría de Educación Pública; inició labores en el Palacio de Bellas Artes en abril de 1945. El objetivo principal de esta escuela era "[...] capacitar al personal que trabajaba en bibliotecas y proporcionarles las técnicas mínimas para desarrollar su trabajo, y que éste se viera reflejado en una mejor organización de las bibliotecas y en adecuados servicios a la comunidad [...]".

Al mismo tiempo se buscaba un mejor salario y reconocimiento profesional, más tarde la escuela tuvo diversos cambios en su estructura, plan de estudios y nombre, y se transformó en lo que actualmente es la Escuela Nacional de Biblioteconomía y Archivonomía [ENBA]..$^{10}$

\section{El Archivo General de la Nación (AGN)}

En definitiva, el tercer congreso de bibliotecarios y primero de archivistas, antes mencionado, representó un hito en la archivística mexicana, pues no sólo propició la creación de la ENBA, sino también permitió al AGN iniciar una serie de acciones tendientes a la regulación de los archivos. Destacan los esfuerzos de Julio Jiménez Rueda para vincular los archivos

7 Ibidem, p. 47.

8 Ibidem, p. 56.

9 Morales Campos, Estela, Educación bibliotecológica en México, México, p. 41.

10 Sotelo, op. cit., p. 18. 
históricos con los administrativos y normalizar las labores del personal y del funcionamiento de los archivos. ${ }^{11}$

Otro de los frutos de este primer congreso fue la expedición de dos leyes, publicadas en el Diario Oficial de la Federación; la primera, en marzo de 1944, facultaba al AGN para determinar el carácter histórico de los documentos, prohibiendo su salida del país. La segunda, publicada el 24 de agosto de ese año, es la llamada Ley de Bienes Nacionales la cual tipifica dentro de los bienes públicos, entre otros, a los expedientes de las oficinas y archivos públicos, los libros raros, las piezas históricas o arqueológicas, las obras de arte de los museos, etc. ${ }^{12}$ La citada ley sería base fundamental de los trabajos que en materia de normatividad archivística se realizaron con posteridad.

El reglamento del AGN de 1946, que sustituyó al de 1920, pretendió también la integración de una comisión consultiva del AGN ésta tendría por objetivo estudiar las medidas tendientes a la modernización de los archivos. En el informe que presentó Jiménez Rueda al finalizar su gestión al frente del AGN en 1952, dejaba claro que para cumplir el objetivo relacionado con el rescate de los archivos debía crearse un órgano autónomo (la Dirección General de Archivos Nacionales) argumentando que, al depender el AGN de la Secretaría de Gobernación, predominaban los intereses políticos sobre los culturales e históricos. ${ }^{13}$

\section{La Universidad Nacional Autónoma de México}

En este contexto en 1952, José María Luján, subdirector de la Biblioteca Nacional, solicitó a las "autoridades universitarias que se establecieran en la Facultad de Filosofía y Letras, cursos de Biblioteconomía y Archivonomía”. ${ }^{14}$

11 AgN, Los Archivos Administrativos en México, pp. 22-25. Véase: Gómez Gómez, Alma Leticia y Torres Monroy, Luis, en "La Necesidad de una Ley Nacional de Archivos", ponencia presentada en X Congreso Nacional de Archivos. Los archivos y la reforma del Estado. Organizado por el Archivo General de la Nación y el Archivo General del Estado de Campeche. Campeche, 28 de octubre de 1998. p. 4.

12 Ibidem, p. 4.

13 Ibidem, p. 5. A partir de 1958, la Secretaría de la presidencia sería la encargada de la reforma administrativa del Estado y, consecuentemente, de la cuestión archivística.

14 Perales de Mercado, Biblioteconomia y Archivonomía en la Universidad Nacional, p. 12. 
Propósito que no era nuevo pues desde 1910, cuando se fundó la Escuela Nacional de Altos Estudios, la Universidad Nacional contempló a la archivística como una disciplina que se tenía que cultivar.

Aunque siempre se hicieron esfuerzos por cumplir con este objetivo, se realizaron acciones concretas en 1956 con la creación de un programa de maestría en archivonomía. ${ }^{15}$ En 1963 la maestría se convirtió en licenciatura y en 1967 cambió su denominación a archivología. La entidad académica responsable era la Facultad de Filosofía y Letras, con dicho plan de estudios se pretendía:

[...] la preparación de profesionistas capaces de planear, organizar y manejar los archivos tanto de tipo histórico como administrativo, nacionales o particulares, ya que éstos son elementos indispensables para el conocimiento del pasado e imprescindibles para la regulación de las actividades de una sociedad [...]

El archivo es [...] un elemento básico de los servicios de información sin los que ninguna actividad puede ser realizada con acierto. Los licenciados en archivología son los profesionistas capaces y preparados para cumplir en la forma más eficiente con este propósito. ${ }^{16}$

En abril de 1975 se suspendió la impartición de la licenciatura debido a que decayó la inscripción, especialmente porque las expectativas laborales eran reducidas, pues las plazas eran ocupadas por personas con formación en otros campos, como la historia, o sin ninguna preparación archivística. ${ }^{17}$

No obstante, la situación ha cambiado pues en la actualidad los especialistas en archivos se colocan rápidamente en posiciones laborales relacionadas con su profesión.

Por otra parte, la disciplina no se ha consolidado debido a diversos factores, entre ellos: un incipiente desarrollo de la archivística mexicana, una limitada conformación de cuerpos de investigación, la falta en el país de

15 En esa época la Facultad de Filosofía y Letras no otorgaba títulos de licenciatura, solamente impartía estudios de maestría que eran equivalentes pues los alumnos ingresaban después del bachillerato. Más tarde, los planes y programas de maestría se convirtieron en licenciaturas y se homologaron con el resto de los planes y programas de estudio de la universidad.

16 Organización académica 1974. Facultad de Filosofía y Letras de la UNAM, p. 57.

17 Sotelo Linares, "Panorama de la enseñanza de la Bibliotecología en la UNAM. Informe Académico por elaboración comentada de material didáctico para apoyar la docencia”, p. 24. 
posgrados en la disciplina y que los archivólogos no han logrado colocarse en los espacios clave dentro de las instituciones y organizaciones, desde donde se podrían impulsar proyectos y actividades que favorecieran la consolidación de la profesión.

\section{Situación actual de la formación profesional}

En la actualidad existen varias instituciones de educación superior que imparten programas formales para el manejo de los archivos y ofrecen opciones de formación a nivel profesional y de especialización o posgrado.

En el afán de ampliar la información para construir un plan de estudios en el campo, se realizó una revisión de los programas que se ofrecen y las diferentes opciones formativas que contemplan. A continuación se exponen los resultados obtenidos de la revisión de programas internacionales y nacionales.

\section{Programas internacionales}

Para su revisión se exploró, en primera instancia, la oferta europea y norteamericana y, luego, los planes que ofrecen universidades de España, Francia, Canadá y Estados Unidos de América. En el cuadro siguiente se describen instituciones que imparten los estudios y los títulos o grados que otorgan:

\begin{tabular}{cll}
\hline \multicolumn{1}{c}{ País } & \multicolumn{1}{c}{ Institución } & Título o grado otorgado \\
\hline \multirow{3}{*}{ Canadá } & University of British Columbia & $\begin{array}{l}\text { Master of Archival Studies } \\
\text { Dual Master of Archival Studies and } \\
\text { Master of Library and Information } \\
\end{array}$ \\
\cline { 2 - 3 } & & Studies \\
\cline { 2 - 3 } & Université de Montréal & $\begin{array}{l}\text { Undergraduate Certificate in Archival } \\
\text { Studies }\end{array}$ \\
\hline
\end{tabular}




\begin{tabular}{|c|c|c|}
\hline País & Institución & Título o grado otorgado \\
\hline \multirow{4}{*}{ España } & Universidad de Sevilla & $\begin{array}{l}\text { Tres especialidades: paleografía, } \\
\text { archivística y biblioteconomía. } \\
\text { Dirigido a graduados en historia, } \\
\text { historia del arte, biblioteconomía y } \\
\text { documentación }\end{array}$ \\
\hline & $\begin{array}{l}\text { Universidad Carlos III de } \\
\text { Madrid. }\end{array}$ & $\begin{array}{l}\text { Máster oficial en archivística y gestión } \\
\text { de documentos }\end{array}$ \\
\hline & $\begin{array}{l}\text { Universidad Internacional de } \\
\text { Andalucía }\end{array}$ & $\begin{array}{l}\text { Máster propio en gestión documental } \\
\text { y administración de archivos. Pueden } \\
\text { acceder quienes tengan título de } \\
\text { licenciado, graduado o diplomado }\end{array}$ \\
\hline & $\begin{array}{l}\text { Universidad Autónoma de } \\
\text { Barcelona }\end{array}$ & $\begin{array}{l}\text { Máster oficial en archivística y gestión } \\
\text { de documentos. Pueden acceder } \\
\text { quienes tengan título de licenciado, } \\
\text { graduado o diplomado }\end{array}$ \\
\hline \multirow{5}{*}{$\begin{array}{l}\text { Estados } \\
\text { Unidos de } \\
\text { América }\end{array}$} & San Jose State University & $\begin{array}{l}\text { Master of Archives and Records } \\
\text { Administration. Online }\end{array}$ \\
\hline & University of Maryland & History/Library Science \\
\hline & Long Island University & Archives \& Records Management \\
\hline & New York University & $\begin{array}{l}\text { M.A. in Archives and Public History } \\
\text { with a concentration in Archives }\end{array}$ \\
\hline & Loyola University Chicago & $\begin{array}{l}\text { Graduate Doctorate } \\
\text { Undergraduate. Bachelor. Master: } \\
\text { - Social Studies and History } \\
\text { - Public History and Archival } \\
\text { Administration }\end{array}$ \\
\hline Francia & École Nationale des Chartes $^{18}$ & $\begin{array}{l}\text { - Le Diplôme d'Archiviste } \\
\text { Paléographe } \\
\text { - Master Technologies Numériques } \\
\text { Appliquées àl'Histoire }\end{array}$ \\
\hline
\end{tabular}

Cuadro 1. Programas de estudios enfocados a los archivos en norteamérica y Europa. ${ }^{19}$

$18 \mathrm{La}$ escuela más antigua de archivística en el mundo, fundada en 1821 en París "para entrenar a los historiadores y a los funcionarios de los archivos en el examen crítico de las fuentes", p. 54.

19 La información de este cuadro se obtuvo de los portales de las distintas instituciones que se refieren. 
Como se puede apreciar, hay una clara tendencia a favorecer la especialización. El requisito previo para el ingreso a estas opciones es un título universitario en cualquier disciplina. En contraparte, en el ámbito latinoamericano, el conocimiento sobre archivística y la preparación de sus profesionales abarcan desde el nivel técnico hasta el profesional.

A partir de la muestra de siete países de Latinoamérica se elaboró el siguiente cuadro:

\begin{tabular}{|c|c|c|c|}
\hline País & Institución & $\begin{array}{c}\text { Título o grado } \\
\text { otorgado }\end{array}$ & $\begin{array}{c}\text { Años en los que se } \\
\text { cursa }\end{array}$ \\
\hline Argentina & $\begin{array}{l}\text { Universidad Nacional } \\
\text { de Córdoba. } \\
\text { Facultad de Filosofía } \\
\text { y Humanidades. } \\
\text { Escuela de } \\
\text { Archivología }\end{array}$ & $\begin{array}{l}\text { Licenciado en } \\
\text { archivología }\end{array}$ & $\begin{array}{l}5 \text { años (con un } \\
\text { título intermedio de } \\
\text { archivero en } 3 \text { años) }\end{array}$ \\
\hline Brasil & $\begin{array}{l}\text { Universidade Federal } \\
\text { Fluminense de Niteró; } \\
\text { Río de Janeiro. } \\
\text { Licenciatura de } \\
\text { Arquivología }\end{array}$ & $\begin{array}{l}\text { Licenciado de } \\
\text { arquivología } \\
\text { Arquivista }\end{array}$ & $\begin{array}{l}4 \text { años bachiller (8 } \\
\text { semestres) }\end{array}$ \\
\hline Colombia & $\begin{array}{l}\text { Universidad de } \\
\text { La Salle. Facultad } \\
\text { de Sistemas de } \\
\text { Información y } \\
\text { Computación }\end{array}$ & $\begin{array}{l}\text { Profesional en } \\
\text { sistemas de } \\
\text { información, } \\
\text { bibliotecología y } \\
\text { archivística }\end{array}$ & 5 años \\
\hline Costa Rica & $\begin{array}{l}\text { Universidad de } \\
\text { Costa Rica. Facultad } \\
\text { de Ciencias } \\
\text { Sociales. Escuela de } \\
\text { Historia. Sección de } \\
\text { Archivística }\end{array}$ & $\begin{array}{l}\text { Licenciado en } \\
\text { archivística }\end{array}$ & $\begin{array}{l}4 \text { años (con un } \\
\text { título intermedio } \\
\text { de bachiller en } \\
\text { archivística, } 3 \text { años) }\end{array}$ \\
\hline Uruguay & $\begin{array}{l}\text { Universidad de la } \\
\text { República. Escuela } \\
\text { Universitaria de } \\
\text { Bibliotecología y } \\
\text { Ciencias Afines. } \\
\text { Licenciatura de } \\
\text { Archivología }\end{array}$ & $\begin{array}{l}\text { Licenciado en } \\
\text { Archivología }\end{array}$ & 3 años \\
\hline
\end{tabular}




\begin{tabular}{clll}
\hline País & \multicolumn{1}{c}{ Institución } & \multicolumn{1}{c}{$\begin{array}{c}\text { Título o grado } \\
\text { otorgado }\end{array}$} & $\begin{array}{c}\text { Años en los que se } \\
\text { cursa }\end{array}$ \\
\hline \multirow{3}{*}{ Perú } & $\begin{array}{l}\text { Universidad Católica } \\
\text { Sede Sapientiae } \\
\text { (UCSS) }\end{array}$ & $\begin{array}{l}\text { Licenciado en } \\
\text { Archivística y Gestión } \\
\text { Documental }\end{array}$ & 5 años \\
\hline
\end{tabular}

Cuadro 2. Programas de estudios enfocados a los archivos en Latinoamérica.

A través de la información proporcionada en el cuadro anterior es posible apreciar una fuerte tendencia a realizar programas de licenciatura con la posibilidad de obtener un título intermedio.

\section{Programas nacionales}

En México hay dos instituciones que imparten licenciaturas en archivos, la ENBA y la Universidad Autónoma de San Luis Potosí (UASLP). Hay una tercera institución que ofrece un programa que se vincula de manera tangencial con los archivos: la Universidad Autónoma del Estado de México (UAEM).

En la ENBA, cabe mencionar que el plan de estudios para la licenciatura en archivonomía se cursa en dos ciclos de formación profesional: el primero de ellos se imparte del $1^{\circ}$ al $5^{\circ}$ semestre que corresponden a la formación del profesional asociado y el segundo, el cual cubre otros cuatro semestres para un total de nueve y otorga el título de licenciado en archivonomía. ${ }^{20}$

La licenciatura en archivología que imparte la UASLP se desarrolla en ocho semestres orientados a la formación de un profesional de la información en las áreas de planeación, administración y gestión de sistemas integrales de archivos.

Finalmente, en lo referente a la licenciatura en ciencias de la información documental que imparte la UAEM, se advierte que las disciplinas archivística, bibliotecológica y la relativa al manejo de la información documental, se entrelazan a lo largo de la carrera. ${ }^{21}$

20 Plan de Estudios de la Licenciatura en Archivonomía, Escuela Nacional de Biblioteconomía y Archivonomía, s.f., http://www.enba.sep.gob.mx/htdocs/files/plan_licenciado_archivo. pdf Consultado el 24 de octubre de 2012.

21 Oferta académica de la Facultad de Humanidades de la Universidad Autónoma del Estado de México, s.f. http://www.uaemex.mx/fhumanidades/oferta/licenciatura.html Consultado el 24 de octubre del 2012. 
Cabe mencionar que la investigación en archivística en las tres instituciones es incipiente, debido al reducido número de profesores con posgrado, la carencia de líneas y proyectos de investigación y la falta de programas de posgrado de la disciplina en México.

\section{Fundamentación de la propuesta}

El proyecto de creación de la licenciatura en administración de archivos y gestión documental respondió a diversos factores: en el aspecto laboral, a la falta de profesionales con el perfil que requiere la administración y gestión de documentos; en el orden económico, a la reducción de costos que significa para las organizaciones contar con personal capacitado para administrar sus archivos; en el orden social, al escaso reconocimiento profesional que tiene la actividad archivística y, en el orden cultural, a la necesidad de consolidar una disciplina de amplia tradición.

El nombre que se da a la licenciatura es un elemento importante pues está sustentado en los siguientes conceptos:

La administración de archivos "como centros [o entidades que] se crean ligados a las instituciones, organismos, familias o personas, públicas o privadas". ${ }^{22}$ De su competencia es también la elaboración de los instrumentos normativos que deben regir su funcionamiento en el ciclo de vida de los documentos (trámite, concentración e histórico).

Por su parte, la gestión documental refiere: "los documentos de archivo se producen naturalmente, inevitablemente, como testimonio y como prueba de la gestión de una institución, familia o persona, y por acumulación van formando el fondo documental, que en algunos casos constituirá el contenido documental del Archivo de la referida institución". ${ }^{23}$

La pertinencia de formar profesionales en este campo responde a una necesidad de alcance nacional, pues se requieren para administrar archivos de instituciones y organizaciones públicas, privadas o sociales y de sujetos particulares.

22 Sotelo Linares, op. cit., p. 23.

23 Antonia Heredia Herrera, p. 23. 
Los egresados deberán:

- Administrar estratégicamente los archivos con un enfoque centrado en la calidad.

- Gestionar los documentos de archivo para hacer accesible la información a los usuarios.

- Promover ante las áreas de informática las mejoras que contribuyan a hacer más eficiente la transmisión de la información.

- Favorecer las condiciones para el cumplimiento de la LFA, cuyo objetivo es:"establecer las disposiciones que permitan la organización y [...] la conservación del patrimonio documental de la Nación”, y de la LFTAIPG que tiene por objetivo "garantizar el acceso de toda persona a la información en posesión de los Poderes de la Unión, los órganos constitucionales autónomos o con autonomía legal, y cualquier otra entidad federal."

- Aplicar cualquier normativa nacional e internacional.

Consideramos que estos propósitos no se cumplen cabalmente en la formación archivística tradicional y responden a imperativos de carácter económico y cultural no considerados hasta ahora.

Los imperativos de carácter económico obligan a la formación de profesionales con conocimientos amplios en los campos del saber señalados, quienes usualmente no poseen la mayor parte de las personas que administran archivos.

Los imperativos de carácter cultural demandan a los licenciados en administración de archivos y gestión documental el aprovechamiento de los sistemas informáticos e identificación de las necesidades de información de los interesados para orientar y brindar las facilidades en la utilización de los documentos lo cual favorece la autenticidad, integridad, fiabilidad y disponibilidad de los documentos.

\section{Estado actual y tendencias futuras}

La identidad de una profesión se basa en un corpus exclusivo de conocimientos y en una cultura profesional, ésta surge de una historia y un propósito 
común, un vocabulario compartido y valores colectivos, así como normas y estándares. El conocimiento básico de los archivistas, por lo tanto, debe abarcar tres aspectos, relacionados entre sí:

a) El conocimiento de la profesión y la evolución de la práctica archivística, las bases teórico-prácticas de la administración, además de referencias contextuales y del marco legal de la profesión.

b) El conocimiento de los documentos de archivo (teoría y metodología asociadas a áreas específicas del trabajo de archivos).

c) El conocimiento del contexto en el cual los documentos se producen, administran y preservan.

La publicación de las leyes federales de transparencia y de archivos ha incrementado la importancia de la profesionalización del personal. La demanda de formación se enfoca fundamentalmente a cursos de capacitación para archivos de trámite y concentración y, en menor grado, a los estudios de licenciatura.

Actualmente no hay suficientes profesionales en archivística que puedan cubrir las demandas surgidas de una sociedad cada vez más globalizada, con nuevos esquemas y retos de trabajo, donde la eficiencia, la eficacia, la calidad, la competencia y el cumplimiento de las obligaciones, son requisitos indispensables. Por ello, la formación de un licenciado en administración de archivos y gestión documental se presenta como una respuesta pertinente a las condiciones del entorno.

Algunos datos más confirman la conveniencia de ampliar las opciones para formar profesionales en archivos, por ejemplo, de acuerdo con el Diagnóstico sobre la situación archivistica de las dependencias y entidades de la Administración Pública Federal, (IFAI): 2007 una cuarta parte de ellas carecía de un programa explícito de capacitación y asesoría en materia archivística. Según el Informe Experto del Banco Mundial (que se publicó en 2000 con el título Los arcbivos de América. Informe experto de la Fundación Histórica Tavera) sobre archivos, en las instituciones encuestadas, $40 \%$ declaró carecer de personal con formación en este campo. ${ }^{24}$

Conviene aclarar también que la ausencia de estos profesionales se ha sustituido parcialmente con programas de capacitación emprendidos por el AGN y el IFAI, dirigidos en su mayoría al ámbito de la administración pública 
federal. Asimismo, desde finales de la década de los ochenta del siglo pasado, instituciones como la Universidad Iberoamericana, la Universidad Nacional Autónoma de México y la Secretaría de Salud han organizado diplomados y cursos encaminados principalmente a la capacitación del personal que labora en los archivos históricos. En tanto, la Red Nacional de Archivos de Instituciones de Educación Superior (RENAIES), a partir de 2004, ha dado diplomados sobre archivos de trámite, concentración e histórico.

De manera paralela, en diversos foros académicos, la comunidad archivística de México ha puesto de manifiesto la necesidad de contar con programas de educación continua para quienes se ven ante la tarea de organizar los documentos que generan y acumulan las dependencias.

También se debe contemplar que los egresados deberán estar en formación permanente, con la posibilidad de ingresar al posgrado. Es importante mencionar que en la administración de archivos y la archivística existe un potencial amplio de temas y líneas de investigación, las cuales posibilitan el desarrollo de proyectos pertinentes y originales, que ayudarán a resolver problemas en las unidades de archivo.

\section{Características de la propuesta}

Con la información analizada se construyó la fundamentación académica para un plan de estudios y se elaboró una propuesta curricular. La carrera se cursaría en ocho semestres, siendo los primeros cuatros de formación básica, donde se darían las herramientas formativas esenciales. Luego vendrían dos semestres de formación intermedia (quinto y sexto), en los semestres séptimo y octavo los estudiantes podrán realizar un proceso de especialización, que en la presente propuesta se llama profundización.

Conviene mencionar que como la propuesta parte del proyecto elaborado conforme al modelo educativo y la estructura de la Escuela Nacional de Estudios Superiores Morelia de la UNAM, de reciente creación, se consideraron los aspectos siguientes:

24 Palos Ramos, Juan José, Aplicación de las tecnologías de información en archivos históricos: proyecto del Archivo Histórico de Monterrey, 18 pp. Disponible en:

http://www.agn.gob.mx/menuprincipal/archivistica/reuniones/2003/rna/pdf/0016.pdf Consultada el 20 de octubre de 2013. 
- Asignaturas por bloques, que deben tener una relación evidente.

- Asignaturas integradoras a lo largo de todos los semestres, para propiciar la interdisciplinariedad.

- El inglés como asignatura obligatoria deibido a que la bibliografía en buena medida está escrita en ese idioma.

- Áreas de profundización que posibiliten al alumno construir su perfil profesional.

- Una opción técnica que facilita al alumno egresar como técnico profesional después del segundo año con la certificación para incursionar en el ámbito profesional.

La estrategia de aprendizaje principal es el estudio basado en problemas, en casos y en proyectos, que en lo general obliga a la construcción de aprendizajes significativos al conectar lo aprendido en el aula con una práctica continua y la aplicación del conocimiento en espacios reales.

En consonancia con el modelo referido en el párrafo anterior, se busca la creación de espacios propicios para el aprendizaje, el profesor asume un papel de orientador, con un fuerte énfasis en vincular los conocimientos teóricos con la práctica.

El objetivo del plan de estudios es formar profesionales capaces de administrar archivos con una visión estratégica, por ello deben recibir formación sobre la teoría y la práctica archivística, la administración, y como elemento integrador, aspectos de las humanidades y ciencias sociales.

También se deben considerar los perfiles, pues permiten identificar las características del sujeto en formación, desde su ingreso hasta el egreso.

En el caso del perfil de ingreso sería deseable que los estudiantes provinieran del bachillerato en ciencias sociales, administrativas o humanidades.

Con respecto al egreso se espera del profesional que sea capaz de:

- Diseñar estrategias administrativas para las unidades de archivo.

- Dirigir proyectos archivísticos de cualquier índole.

- Incidir en la toma de decisiones.

- Intervenir en las características de la producción de los documentos.

- Gestionar documentos en cualquier soporte. 
- Elaborar políticas, manuales y reglamentos archivísticos.

- Capacitar al personal de las áreas de archivos.

- Evaluar archivos.

La propuesta incluye dos áreas de profundización que se cursan durante el cuarto año de la carrera y cuyo objetivo es especializar al estudiante a partir de la formación integral de los tres primeros años.

Se plantea un área de especialización en valoración documental, espacio profesional en donde existe escasez de expertos capaces de realizar esta tarea y que hayan recibido preparación universitaria. Los egresados que elijan esta área de profundización serán capaces de:

- Identificar los atributos de los documentos para establecer su valor de conservación.

- Diseñar e implementar diversos instrumentos archivísticos para justificar y formalizar la disposición documental.

- Coordinar equipos de trabajo para efectuar la valoración y disposición de documentos.

Una segunda área de profundización es archivos históricos, en el caso de México es realmente pertinente por la cantidad de documentos con esas características que necesita ser gestionada y administrada por un profesional capaz de:

- Evidenciar el alcance histórico de los archivos.

- Rescatar testimonios con valor histórico.

- Organizar y describir archivos históricos.

También se contempla en el quinto semestre de la carrera, el egreso del estudiante como técnico profesional en archivos, quien adquirirá conocimientos para:

- Aplicar acciones de rescate, preservación, identificación, valoración, selección, organización, descripción, uso y disposición de los documentos localizados en los archivos. 
- Identificar los elementos centrales de la gestión y la administración, con énfasis en la innovación administrativa.

Por otra parte para el egresado como licenciado y técnico profesional se consideran como espacios laborales potenciales:

- Las secretarías federales y estatales

- Los gobiernos municipales

- Las cámaras del poder legislativo federal o estatal

- Los distintos órganos del poder judicial

- Los organismos públicos autónomos

- Las instituciones educativas

- Los institutos de transparencia

- Los archivos históricos federales, estatales y municipales

- Las empresas

- Los organismos internacionales

- Las instituciones educativas

- Las instituciones religiosas

- Los organismos no gubernamentales

La licenciatura en administración de archivos y gestión documental se cursará en ocho semestres con 68 asignaturas.

La opción técnica profesional tendrá una duración de cinco semestres. Los primeros cuatro son comunes. El alumno que elija esta opción cursará una asignatura adicional llamada práctica supervisada, única a cursar en el quinto semestre y cuyo objetivo es obtener el título correspondiente.

Como ya se mencionó el plan de estudios tiene dos etapas formativas. La primera abarca del primero al cuarto semestre, incluyendo 35 asignaturas; de las cuales 25 pertenecen a la parte formativa, dos son optativas, cuatro talleres de integración, así como cuatro de aprendizaje del idioma inglés.

La segunda etapa, a partir del quinto semestre, comprende los últimos cuatro semestres y tiene un total de 33 asignaturas: de las cuales 22 corresponden a las áreas de profundización; cuatro son talleres de integración, cuatro son asignaturas de inglés y tres asignaturas optativas.

Por las características de la licenciatura, las prácticas son medulares 
para la formación de los alumnos, constituyen un balance con la teoría de la administración de archivos y gestión documental, así como con las humanidades y ciencias sociales. Por lo anterior, todas las asignaturas tienen un componente teórico y práctico. Una parte práctica se desarrolla en cada curso y otra se concentra en los talleres de integración que se efectúan al final de cada semestre y que favorece la reflexión, la aplicación de conocimientos y la elaboración de productos académicos.

El plan de estudios establece mecanismos de flexibilidad que facilitan la organización curricular que se explica a continuación:

- Solo se propone seriación indicativa en los talleres de integración y en las asignaturas de inglés.

- A lo largo de la licenciatura, es fundamental la actividad tutorial, especialmente en los dos primeros años.

- El alumno podrá elegir las asignaturas optativas de cualquier licenciatura que se imparta en la facultad o escuela.

En la organización del plan de estudios se establecen tres campos de conocimiento vinculados en el proceso formativo e integral del alumno:

- Teoría, metodología y práctica archivística

- Administración y sistemas

- Humanidades y ciencias sociales

\section{Teoría, metodología y práctica archivística}

Este primer campo está integrado por asignaturas que articulan el pensamiento abstracto derivado de la teoría archivística, el cual se vinculará con el análisis de los procesos de gestión de documentos y administración de archivos, asimismo se consideran asignaturas pertenecientes a otras disciplinas del conocimiento y que son necesarias para la preservación, conservación, reprografía y difusión de los documentos.

Los estudiantes cursarán asignaturas cuyo propósito es analizar los conceptos acerca de los procesos archivísticos de identificación, clasificación, ordenación, descripción, recuperación de la información y 
la evolución de la estructura interna y externa de los tipos documentales producidos por las instituciones mexicanas. Del mismo modo, distinguir las áreas que desarrollan funciones de recepción y despacho de correspondencia con el fin de gestionar la producción de documentos de las entidades.

Además, el estudiante tendrá un acercamiento a las áreas de profundización correspondientes a los semestres séptimo y octavo, para desarrollar un trabajo archivístico complejo en escenarios reales.

\section{Administración y sistemas}

El segundo campo de conocimiento contempla aspectos tales como planeación, organización, control y supervisión de los recursos involucrados en el archivo de una institución. En este sentido se concibe que el profesional sea quien dirija los procesos archivísticos, además de planear, difundir y promover servicios acordes con las necesidades de los usuarios.

Asimismo, se pretende que él tenga la capacidad para atender las necesidades de recursos humanos, financieros y materiales, requeridos en los archivos para que la eficiencia y eficacia redunden en áreas operativas con el principio de calidad.

Los archivos por su naturaleza integran el patrimonio cultural de la sociedad, y como indica la Society of American Archivists (SAA):

[...] sin una cuidadosa selección de registros, cada sociedad perderá su patrimonio cultural, comercial, institucional, científico y social. Sin la preservación de los documentos legales, los derechos individuales e institucionales no pueden ser preservados y protegidos, y sin el manejo adecuado de los registros administrativos, los gobiernos y las organizaciones no pueden ser considerados responsables. Los registros mantenidos en los archivos son, por lo tanto, esenciales en una sociedad democrática y educada. ${ }^{25}$

En este campo el alumno adquirirá las habilidades necesarias para la administración de archivos, el diseño y la implementación de sistemas de

25 Guidelines for a Graduate Program in Archival Studies (GPAS). p. 1. Disponible en: http:// www2.archivists.org/book/export/html/69. Consultada el 20 de octubre de 2013. 
información en las instituciones y en las organizaciones, la elaboración de programas de calidad y el desarrollo de proyectos.

En los primeros cuatro semestres el estudiante analizará las características de la cultura organizacional para reconocer su relación con los sistemas de calidad y las variables de la comunicación. Asimismo, adquirirá conocimientos sobre la administración de las instituciones públicas mexicanas en los tres niveles de gobierno con el propósito de identificar las funciones y estructuras de los organismos públicos, utilizará la tecnología en los procesos archivísticos y revisará los fundamentos teórico-metodológicos de las organizaciones para identificar sus características y transformaciones.

Una parte distintiva de la propuesta está en el énfasis en comprender el alcance de los sistemas, por lo que resulta relevante que reconozca las aplicaciones y los programas más acordes para administrar archivos y gestionar documentos. Todos los elementos enunciados llevarán al estudiante a profundizar en los procesos administrativos y en las teorías del liderazgo para su aplicación en la práctica profesional.

También se procura que el estudiante aprenda a organizar los flujos de información documental para su adecuada gestión, a reconocer las bases de la administración de los recursos financieros y materiales para su aplicación en las instituciones y organizaciones, así como analizar los conceptos, procesos y alcance de la planeación estratégica.

En el séptimo y octavo semestre el alumno analizará las variables de la administración de los recursos humanos para mejorar la capacidad laboral; igualmente elegirá una de las dos áreas de profundización para incorporarse al mercado de trabajo con conocimientos sólidos, ya sea en valoración documental o en archivos históricos.

\section{Humanidades y ciencias sociales}

El tercer campo de conocimiento tiene como propósito principal que el alumno cuente con una perspectiva integradora y sea competente para vincular el conocimiento archivístico y administrativo con los saberes históricos, filosóficos, sociológicos, pedagógicos y jurídicos.

La función formativa de las humanidades pretende que el egresado sea capaz de comprender el devenir de las instituciones, lo cual es esencial para 
ubicar en el espacio y el tiempo las prácticas sobre la creación y conservación de los documentos.

Al trabajar con documentos también resulta conveniente que los estudiantes conozcan la historia de la escritura, pues le proporcionará saberes de utilidad para la gestión y la administración.

Por otra parte, la propuesta contempla la asignatura de teoría del conocimiento, con la cual el estudiante recibirá una formación que lo apoyará para comprender las bases teóricas de las disciplinas administrativa y archivística. Otro elemento importante es la ética deontológica, ésta preparará a los estudiantes para consolidar los valores que le permitan asumir las responsabilidades propias de la profesión. Este aspecto responde al deber de reforzar los principios que propicia la preservación de la integridad de cada documento y de su conjunto, porque son la base para la toma de decisiones y para conformar la memoria y el acervo social, histórico y cultural de la sociedad. Además, es vital conocer el fundamento contable, legal y administrativo para respaldar las acciones realizadas.

El responsable de un archivo, que asume como propio el valor ético en la administración y gestión, será un sujeto clave para facilitar el trabajo de auditoría que realizan distintas instancias públicas y privadas. Asimismo, será un agente importante para la transparencia y rendición de cuentas establecidas por la normativa federal.

Con respecto a la formación en ciencias sociales, el conocimiento del marco legal de los archivos brindará a los egresados herramientas para comprender que los procesos de carácter administrativo y archivístico están ligados a una normativa. Además, será sustancial comprender los elementos centrales de la propiedad intelectual, esto en el caso de la documentación tiene especial importancia.

La propuesta también responde a una doble necesidad: capacitar y ejercer la docencia. Por una parte el administrador realizará o facilitará la capacitación del personal que efectúa la gestión. Por la otra, una carrera primordialmente práctica, dará a los egresados conocimientos de estrategias didácticas para ejercer como profesores.

Las humanidades y las ciencias sociales son esenciales para preservar nuestra producción y herencia documental. 


\section{Conclusiones}

En el campo de la administración de archivos y gestión documental las opciones profesionales que se tienen en el país no responden plenamente a las necesidades del mercado laboral, ante el cúmulo de documentos que actualmente generan los sectores público, privado y social, la cantidad de archivos a lo largo y ancho del país, así como a la obligatoriedad establecida en la legislación. De aquí, la necesidad de formar profesionales con bases sólidas para la toma de decisión oportuna frente a la complejidad de los cambios políticos, económicos, sociales y tecnológicos.

La formación de un profesional en la materia requiere conocimientos administrativos, archivísticos, humanísticos y sociales para poseer una visión innovadora, estratégica e integral la cual propicie que los archivos cumplan su misión social, importante para la gestión, la investigación y la preservación de la memoria histórica del país.

Esta propuesta muestra la relevancia de fortalecer la administración de archivos y la disciplina archivística, en aras de optimizar la función de las instituciones y las organizaciones, la democratización de la sociedad y la conservación de los testimonios históricos del país.

\section{Bibliografía}

agn, Los Archivos Administrativos en México, Cuadernos de formación archivística, AGN, núm. 1, [serie Principios básicos de administración de documentos], 1986.

Camacho García, P. E. La experiencia de México en sistemas de información y automatización de archivos. En: Memoria XIV Jornada para el Desarrollo Archivístico (26-27 julio 2001). San José de Costa Rica, Ministerio de Cultura, Juventud y Deportes, Dirección General del Archivo Nacional, 2002.

Diagnóstico sobre la situación archivística de las dependencias y entidades de la administración pública federal: 2007. México: Instituto Federal de Acceso a la Información Pública, 2008.

Echeverría, Javier, “Apropiación social de las tecnologías de la información y la comunicación", en Revista Iberoamericana de Ciencia, Tecnología y Sociedad, 2008, vol. 4, núm. 10 [consultado 05/03/2014]. Disponible en: http:// 
oeibolivia.org/files/Volumen\%204\%20-20N\%C3\%BAmero\%2010/ doss07.pdf

Escuela Nacional de Biblioteconomía y Archivonomía (México). Plan de Estudios de la Licenciatura en Archivonomía, México, ENBA [Consultado 05/03/2014]. Disponible en: http://www.enba.sep.gob.mx/files/plan_ licenciado_archivo.pdf

Escuela Nacional de Biblioteconomía y Archivonomía (México), Plan de Estudios para el Profesional Asociado en Archivonomía, México, ENBA [consultado 05/03/2014]. Disponible en: http://www.enba.sep.gob. $\mathrm{mx} /$ files/plan_asociado_archivo.pdf

Guidelines for a Graduate Program in Archival Studies (GPAS), USA, Society of American Archivists, 2011. [Ccnsultado 28/03/2014]. Disponible en: http://www2.archivists.org/book/export/html/69.

Heredia Herrera, Antonia. ¿Qué es un archivo?, Gijón, Trea, 2007.

Iggers, Georg G. La historiografía del siglo XX: desde la objetividad cientifica al desafío posmoderno, Chile, FCE Chile, 2012.

Iguíniz, Juan Bautista, "Apuntes para la historia de la enseñanza de la Biblioteconomía en México", en Boletin de la Escuela Nacional de Bibliotecarios y Archivistas, México, 1954, vol. 3-4.

Morales Campos, Estela, Educación bibliotecológica en México, México, UNAM, Cuib, 1989, Monografías, núm. 6.

Perales De Mercado, Alicia, "Biblioteconomía y Archivonomía en la Universidad Nacional", en Anuario de biblioteconomía y archivonomía, México, UNAM, FFyL, 1961, 1 a época, núm.1.

Rodríguez Mederos, Mabel; Montes De Oca Sánchez De Bustamante, Antonio; Dorta Héctor, Juliemne. Utilización y conservación de los soportes electrónicos. Acimed, 2002, vol. 10, núm. 6, [consultado 05/03/2014]. Disponible en: http://eprints.rclis.org/5128/1/ utilizacion.pdf

Sotelo Linares, Sandra. "Panorama de la enseñanza de la bibliotecología en la UNAM”, México, UNAM, Facultad de Filosofía y Letras, 2008. Informe Académico para obtener el título de licenciada en bibliotecología [consultado 05/03/2014]. Disponible en: http://www.filos.unam.mx/ LICENCIATURA/bibliotecologia/textos-apoyo-docencia/sotelolinares-sandra.pdf 
Universidad Autónoma del Estado de México, Facultad de Humanidades, [oferta académica], [consultado 05/03/2014]. Disponible en: http:// www.uaemex.mx/fhumanidades/oferta/licenciatura.html

Universidad Nacional Autónoma de México, Facultad de Filosofía y Letras: organización académica, México, UNAM, Dirección General de Orientación Vocacional, 1974.

Universidad Autónoma de San Luis Potosí. Escuela de Ciencias de la Información. Oferta educativa: Licenciatura en Bibliotecología, [consultado 05/03/2014]. Disponible en: http://www.uaslp.mx/Spanish/ Academicas/ECI/OFE/Paginas/default.aspx

Villanueva Bazán, Gustavo, "La Archivística: una ciencia en busca de sí misma (México 1915-1945)", tesis de máster en gestión documental y administración de archivos, Universidad Internacional de Andalucía, España, 2012. D 